\title{
Sign Language Recognition by Finger Vector
}

\author{
Krisada Vilailak
}

\begin{abstract}
The sign language is very important and necessary for deaf since the language is the only way to communicate for deaf and normal people who need to send and receive the messages from the deaf-mute. This paper has suggested a concept of the sign language recognition with finger vectors. A hand figure showing the sign language would be downloaded at runtime using Freeman chaincode attaching on the edge of photos. Then the contour line would appear, detected fingertips and furrows between fingers, that would be transformed to be finger vectors. After that, it could be done by coding and saving in the database in order to compare for recognition further. Moreover, the sign language of 45 samples of number 1-9 were tested, and $98 \%$ of accuracy was found. However, this concept has the prominent point because scaling, rotating, thinning or Noise filtering do not need to be done; therefore, it can save up a lot of processing time.
\end{abstract}

Keywords: Sign language, Finger vector, freeman chaincode

\section{INTRODUCTION}

Sign language is currently the most important form of communication towards deaf-mute. Deaf people contact each other with sign language providing different signs with complex meanings. For normal people, to understand sign language is quite difficult and complicated. It is hard to find experienced sign language translator who can understand it clearly. However, humans prevalently use computer around the world, so the concept using application to help underprivileged people has been created. With this concept, deaf-mute can communicate with normal people.

\section{II.LITERATURE REVIEW}

There have been a lot of sign language studies such as : A different method had been developed by Archana S Ghotkar, Rucha Khatal, Sanjana Khupase, Surbhi Asati and MIthila Hadop through Hand Gesture Recognition for Indian Sign Language consisted of use of Camshift and HSV model and then recognizing gesture through Genetic Algorithm, in the following applying camshift and HSV model was difficult because making it compatible with different MATLAB versions was not easy and genetic algorithm takes huge amount of time for its development [2]. A method had been developed by T. Shanableh for recognizing isolated Arabic sign language gestures in a user independent mode. In this method the signers wore gloves to simplify the process of segmenting out the hands of the signer via color segmentation. The effectiveness of the proposed userindependent feature extraction scheme was assessed by two different classification techniques; namely, K-NN and polynomial networks.

Krisada Vilailak, Electrical Branch, Faculty of Engineering Vongchavalitkul University, Nakornratchasrima , Thailand
Many researchers utilized special devi to recognize the Sign Language[3]. Byung - woo min et al, presented the visual recognition of static gesture or dynamic gesture, in which recognized hand gestures obtained from the visual images on a 2D image plane, without any external devices. Gestures were spotted by a task specific state transition based on natural human articulation[4]. A method had been developed by P Subha Rajan and Dr G Balakrishnan for recognising gestures for Indian Sign Language where the proposed that each gesture would be recognised through 7 bit orientation and generation process through RIGHT and LEFT scan. The following process required approximately six modules and was a tedious method of recognising signs[5].

This research suggested vector technique of fingers which is not complex, and it gives high efficiency.

\section{PROPOSED METHOdOLOGY}

It can be started with input photos showing sign language as numbers into computer, and followed the methods. Figure 1

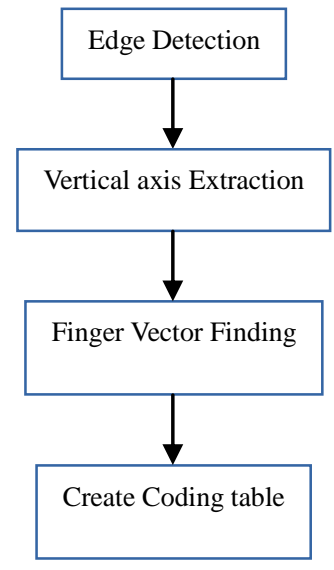

Fig. 1 Recognition Algorithm

Edge Detection : Using Freeman chain code : Chain $\operatorname{code}^{[1]}$ is used to represent a boundary by a connected sequence of straight line segments of specified length and direction. Typically this representation is based on 4 (or) 8 connectivity of the segments (as shown in Fig.1 a-b). The direction of each segment is coded by using a numbering scheme. A boundary code formed as a sequence of such directional numbers is referred to as a Freeman Chain Code. The chain code of a boundary depends on the starting point. Working with code numbers offers a unified way to analyze the shape of the boundary. Chain Code follows the contour in counter clockwise manner and keeps track of the directions as we go from one contour pixel to the next. Fig.1(a) shows 4connectivity and Fig.1(b) shows 8-connectivity of freeman chain code. 


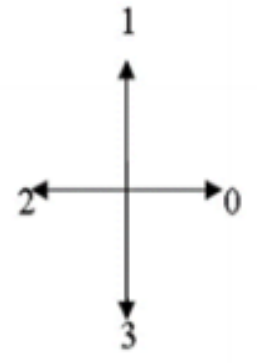

(a)

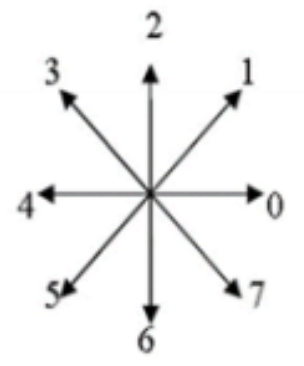

(b)
Fig. 2 Neighbour Directions of Freeman Chain code

Vertical Axis Extraction : dividing chaincode into vertical axis. It determined upward direction to be ' + '; downward direction to be '-'. it determined right or left hand direction to be ' 0 '. Where ' + ' have been changed to be '-', meaning tipping point, but probably not fngertip.

Finger Vector Finding : detemined the middle of bottom of palm as the center point, and drawing a straight line from tipping point to the center in order to find length and angle which is finger vector as shown in Fig.3. Then, using the longest vector as base for grouping every vectors in the same unit with the following equation :

$$
\begin{gathered}
\text { length }_{\text {new }}=\left(\text { length } h_{\text {old }}-\frac{\text { Base }}{2}\right) \times \frac{100}{\text { Base }} \\
\theta=\tan ^{-1} \frac{\left(y_{i}-y_{\text {center }}\right)}{\left(x_{i}-x_{\text {center }}\right)} \times \frac{180}{\pi}
\end{gathered}
$$

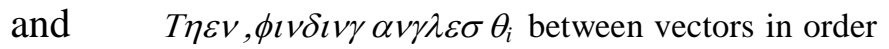
to create coding table.

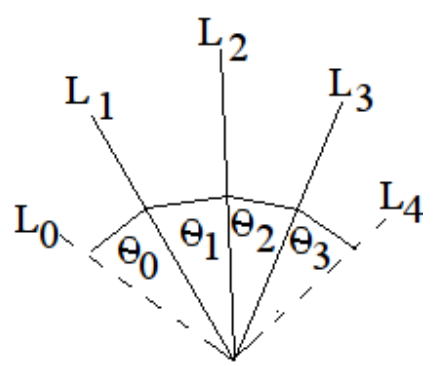

Fig 3. Finger vector

Create Coding Table : Considering finger vector , fingertips and $\theta_{i}$, can create the coding table below.

\begin{tabular}{|c|c|c|c|}
\hline \multicolumn{5}{|c}{ TABLE 1. CODING TABLE } \\
\hline Number & Fingertips & $\begin{array}{c}\text { Rest } \\
\text { finger }\end{array}$ & $\begin{array}{c}\theta_{3}>\theta_{1}+\theta_{2} \\
\theta_{4}>\theta_{2}+\theta_{3}\end{array}$ \\
\hline 1 & 1 & - & no \\
\hline 2 & 2 & - & no \\
\hline 3 & 3 & 1 & yes \\
\hline 4 & 4 & - & no \\
\hline 5 & 5 & - & yes \\
\hline 6 & 3 & 1 & no \\
\hline 7 & 3 & 2 & no \\
\hline 8 & 3 & 3 & no \\
\hline 9 & 3 & 4 & no \\
\hline
\end{tabular}

\section{RESULTS}

The results of recognition in Fig.4(a)-(i) showed.

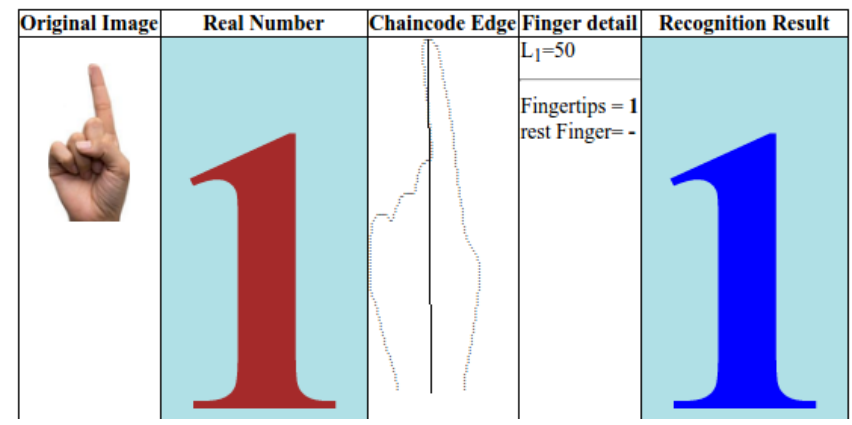

(a)

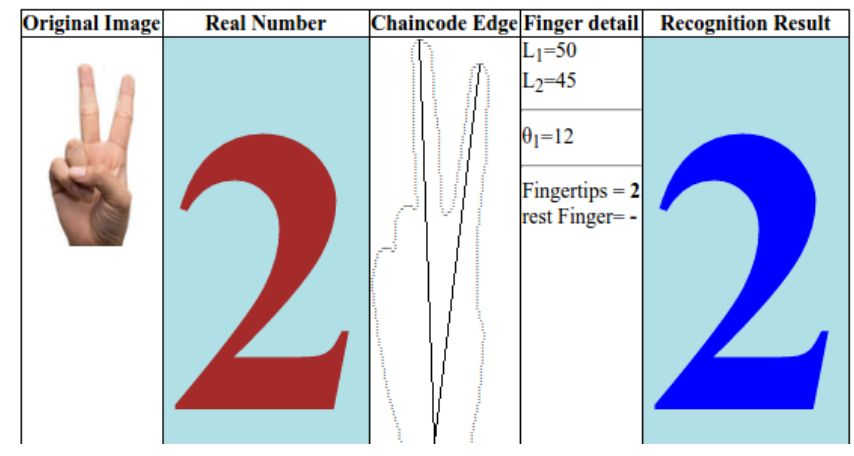

(b)

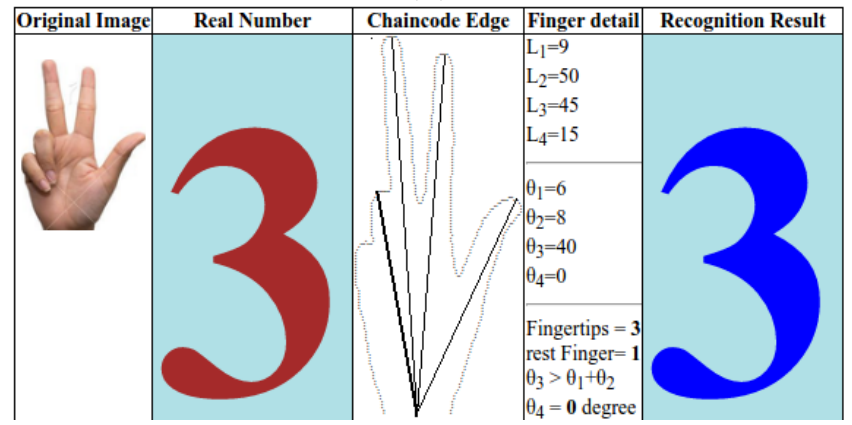

(c) 


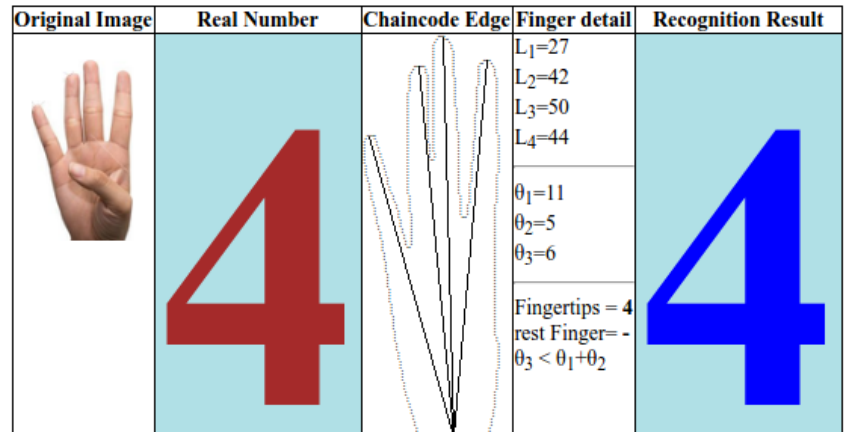

(d)

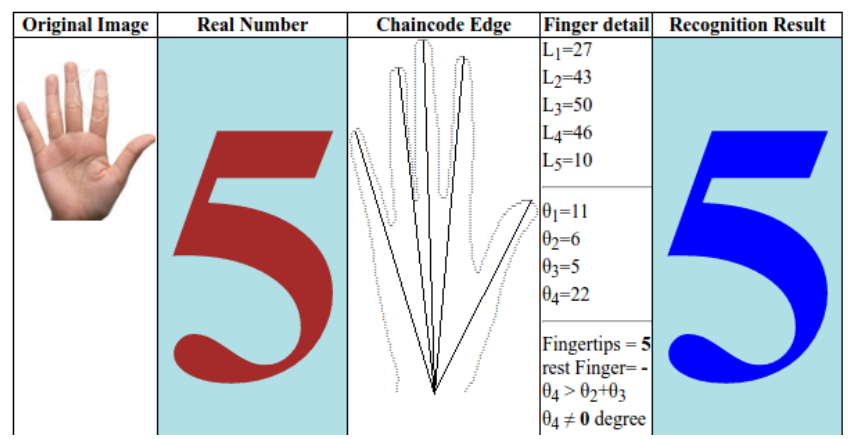

(e)

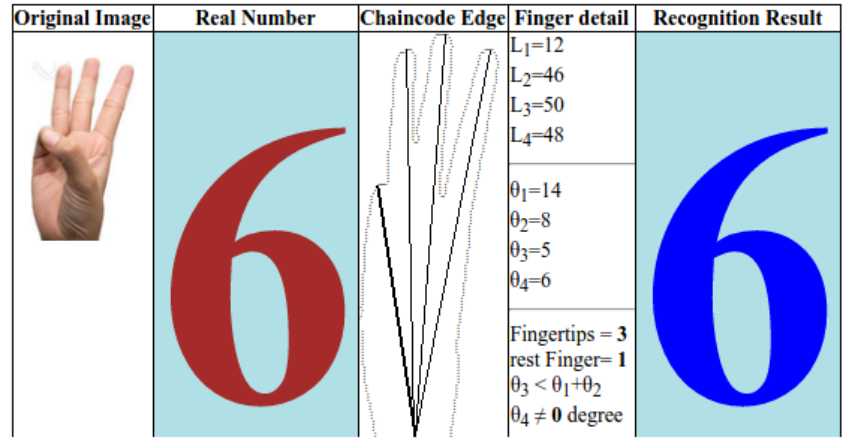

(f)

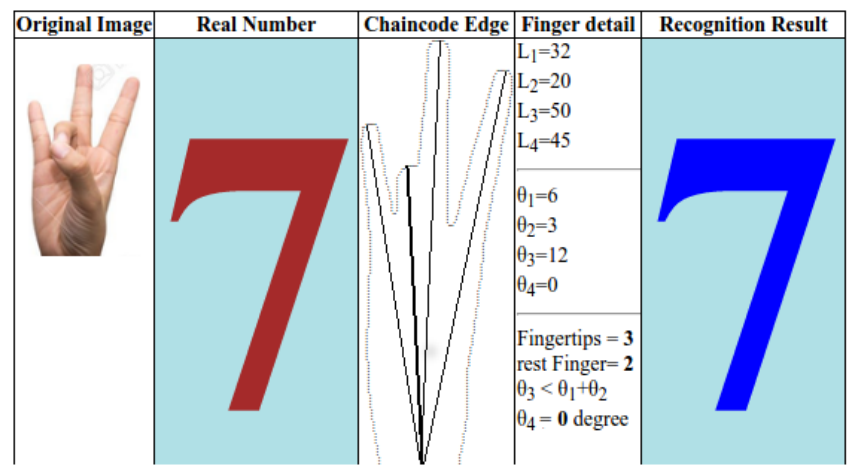

$(\mathrm{g})$

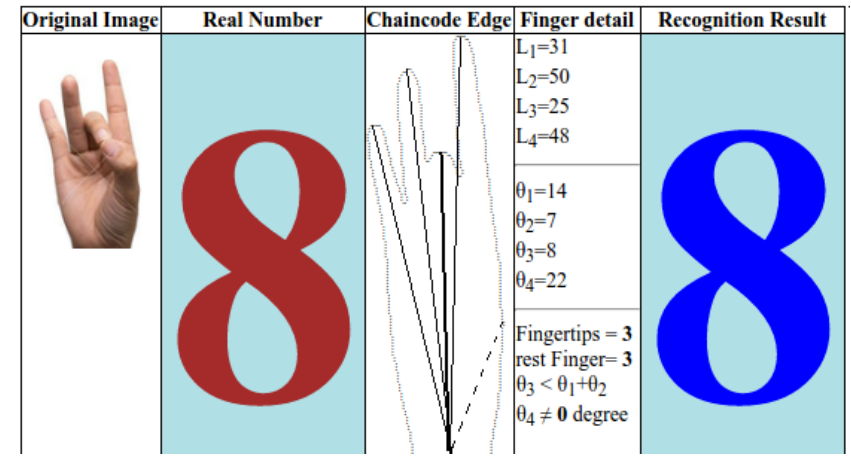

(h)

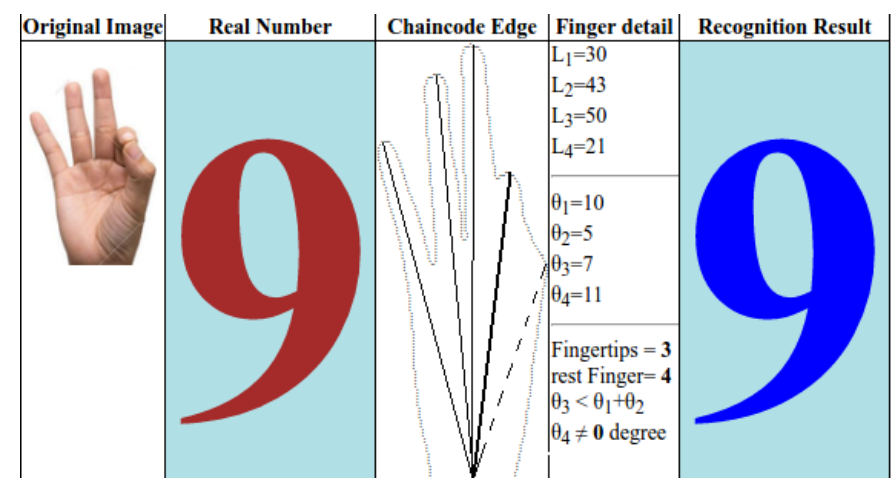

(i)

Fig.4 (a)-(i) Show results of Recognition

\section{CONCLUSION}

The results of recognition in Fig.8(a)-(d) showed input in the system and edge detection with Freeman chain code, and it calculated the numbers of tipping point and extracted tipping or non $\mathrm{g}$ of fingertips. Then, it created verification code and compared to the produced coding table. It can be seen that the results of 45 photos of 9 forms of sign language recognition are $100 \%$ accurate. This concept has the prominent point because scaling, rotating, thinning or Noise filtering do not need to be done.

\section{ACKNOWLEDGEMENT}

Without the research presentation fund of Vongchavalitkul University, this research would not have been possible.

\section{REFERENCES}

[1] Digital Image Processing $2^{\text {nd }}$ Edition by Gonzalez and Woods Pearson Publications

[2] Ghotkar, Archana S., "Hand Gesture Recognition forIndian Sign Language", International Conference on Computer Communication and Informatics(ICCCI), 2012, pp 1-4.

[3] Shanableh, Tamer, T. Khaled, "Arabic sign language recognition in user independent mode", IEEE International Conference on Intelligent and Advanced Systems, 2007, pp 597-600.

[4] Byung-woo min, Ho-sub yoon, Jung soh, Takeshi ohashi and Toshiaki jima," Visual Recognition of Static/Dynamic Gesture: Gesture-Driven Editing System", Journal of Visual Languages \& Computing Volume10,Issue3, June 1999, pp 291-309.

[5] Rajam, P. Subha and Dr G Balakrishnan, "RealTime Indian Sign Language Recognition System to aid Deaf and Dumb people", 13th International Conference on Communication Technology(ICCT), 2011,pp 737-742 